\title{
OPTIMIZATION OF THE LCLS X-RAY FEL OUTPUT PERFORMANCE IN THE PRESENCE OF STRONG UNDULATOR WAKEFIELDS
}

\author{
S. Reiche, UCLA, CA 90095, USA - W.M. Fawley, LBNL, CA 94720, USA \\ K.L.F. Bane, P. Emma, Z. Huang, H.-D. Nuhn, G. Stupakov, SLAC, CA 94309, USA
}

\begin{abstract}
The Linac Coherent Light Source (LCLS) Free-Electron Laser will operate in the wavelength range of 1.5 to 15 Angstroms. Energy loss due to wakefields within the long undulator can degrade the FEL process by detuning the resonant FEL frequency. The wakefields arise from the vacuum chamber wall resistivity, its surface roughness, and abrupt changes in its aperture. For LCLS parameters, the resistive component is the most critical and depends upon the chamber material (e.g. $\mathrm{Cu}$ ) and its radius. To study the expected performance in the presence of these wakefields, we make a series of start-to-end simulations with tracking codes PARMELA and ELEGANT and time-dependent FEL simulation codes Genesis 1.3 and Ginger. We discuss the impact of the wakefield on output energy, spectral bandwidth, and temporal envelope of the output FEL pulse, as well as the benefits of a partial compensation obtained with a slight $\mathrm{z}$ dependent taper in the undulator field. We compare these results to those obtained by decreasing the bunch charge or increasing the vacuum chamber radius. We also compare our results to those predicted in concurrent analytical work.
\end{abstract}

\section{INTRODUCTION}

The Linac Coherent Light Source (LCLS) is a X-ray Free-Electron Laser (FEL), currently under construction at SLAC [1]. It is designed to operate in the wavelength range of 1.5 - 15 Ångstrom. Unlike alternative designs of X-ray FELs (e.g.[2]), the gap of the LCLS undulator modules is fixed and the wavelength is tuned by adjusting the electron beam energy. The vacuum chamber, which fits between the undulator poles, has an inner radius of $2.5 \mathrm{~mm}$, which causes strong undulator wakefields. The change in the electron energy by these wakefields disrupts the FEL performance by pushing electrons off-resonance. Because the wakefields are not constant but depend on the position along the electron bunch, the effect cannot be compensated globally by an adjustment of the undulator field (taper).

In this paper we continue the study of the impact of undulator wakefields on the performance of LCLS [4], where we have extended the model for the wakefields to include the frequency dependence of the conductivity of the vacuum chamber material. The FEL output is optimized by varying the undulator field taper gradient. We consider two cases: the standard case with a bunch charge of $1 \mathrm{nC}$ and an alternative low charge case of $200 \mathrm{pC}$ with reduced wakefields and a more stable operation of the machine [3].

\section{START-END SIMULATIONS AND WAKEFIELD MODEL}

The performance of the LCLS Free-Electron Laser is modeled with a set of specialized simulation codes. The beam dynamics in the rf photo injector and the initial stage of accelerations is done with PARMELA. The phase space distribution is then imported into ELEGANT, which tracks the electron beam through the main linac to the undulator, including the two bunch compressors and the linacundulator transport line, preceding the undulator. The coherent synchrotron radiation effects during compression are modeled with an analytical model within ELEGANT. The two time-dependent FEL codes GINGER and GENESIS 1.3 then calculate the evolution of the FEL pulse, based on the electron beam phase space distribution of the ELEGANT output. For this study, misalignment or errors of beamline components in the injector, linac and undulator are excluded.

Both FEL codes support undulator wakefields and have been used for previous studies of wakefield effects within the LCLS undulator. However the underlying wakefield model for the simulations has been extended to include the AC component of the resistive wall wakefields [5], which adds a larger contribution to the total wakefields for a $\mathrm{Cu}$ vacuum chamber than for an $\mathrm{Al}$ chamber. Thus the former result of wakefield simulations, that $\mathrm{Cu}$ is the preferred choice for the vacuum chamber, became questionable. With the accurate model, the simulation results should yield a more definite answer.

A new aspect in these wakefield simulations is the partial compensation of wakefields by a slight linear taper of the undulator field [6]. With negligible wakefield amplitudes a slight increase in the undulator field strength can yield an enhancement of the FEL power by up to 100 percent. For convenience, the strength of the field taper is expressed by the equivalent energy loss, for which the resonance condition is kept preserved by the taper.

\section{THE STANDARD CASE}

In the improved wakefield model, the frequency dependence of the conductivity is included. Compared to former results [4] a large contribution is added to the wake fields for copper by the frequency dependence of the conductivity. The changes in the wakefields for aluminum are negligible. The accurate wake potential for both materials are shown in Fig. 1. The AC wake introduces an oscillation of the wake potential over the main body of the bunch, 

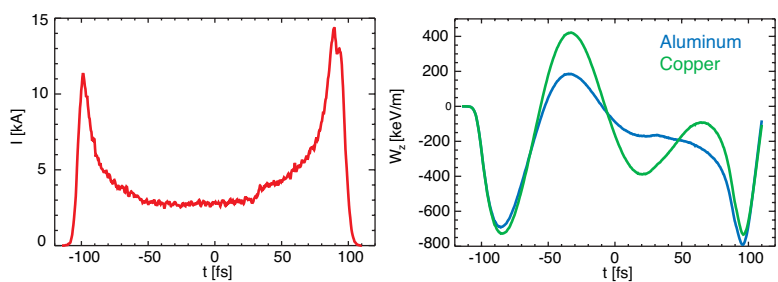

Figure 1: Current profile and undulator wakefields (left and right plot, respectively) for a $1 \mathrm{nC}$ electron bunch charge.

which is damped along the bunch more slowly than the resistive wake potential for aluminum. Therefore copper is no longer preferred as the material of choice because the wake potential is not flat over the main body of the bunch and cannot be fully compensated by a field taper.

The degradation in the FEL output energy is strong for both materials $(0.9 \mathrm{~mJ}$ for aluminum and $0.8 \mathrm{~mJ}$ for copper compared to $2 \mathrm{~mJ}$ in the case when wakefields are excluded). Undulator tapering recovers the energy degradation but does not improve the performance beyond $2 \mathrm{~mJ}$ output power. The optimum taper is $200 \mathrm{keV} / \mathrm{m}$ for aluminum and $300 \mathrm{keV} / \mathrm{m}$ for copper with a rather large tolerance $( \pm 50 \mathrm{keV} / \mathrm{m})$.

Even after the optimization for both materials the radiation pulse envelope is strongly distorted for the case of wakefields and field taper while it is smooth and continuous in the ideal case of excluded wakefields. Varying the field taper will change the envelope but the general fact remains that the pulse is split up into three or four fragments (Fig. 2). This breakup is caused by the oscillation in the wakefield whose amplitude is larger than the FEL tolerance of about $100 \mathrm{keV} / \mathrm{m}$, yielding a more continuous power envelope. Because the wake potential for aluminum is almost constant in the tail of the electron bunch (as seen in the range of 0 fs to $70 \mathrm{fs}$ in the right plot of Fig. 1) it reduces the impact of fragmentation so that at least the last half of the FEL pulse envelope is continuous and uniform in amplitude.

The resistive wall wakefield has a $R^{-2}$ dependence on

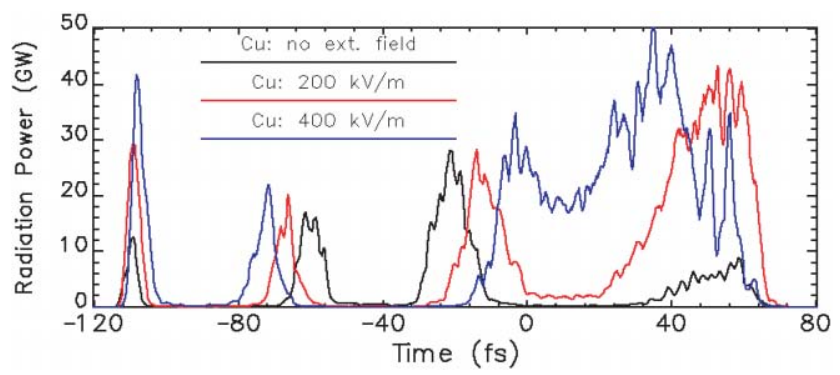

Figure 2: Radiation pulse envelope at the undulator exit for a copper vacuum chamber and various degrees of undulator field taper. the vacuum chamber radius $R$, but in order to increase the radius the gap of the undulator has to be increased as well. This increase yields a reduction in the undulator parameter and the electron beam energy to preserve the resonance condition. The partial benefit of a larger gap is compensated by a lower beam power and the output energy of LCLS remains almost constant for a chamber radius from $2.5 \mathrm{~mm}$ to $5 \mathrm{~mm}$ [7]. Another solution is to change the vacuum chamber aperture to an elliptical cross section with a large aspect ratio. In the limit of two parallel plates the amplitude of the resistive wakefield is reduced by the factor $\pi^{2} / 16$ [8]. Although the maximum amplitude of the wake potential lies still outside of the FEL tolerance of 100 $\mathrm{keV} / \mathrm{m}$, the smaller wakefields reduce the fragmentation of the FEL pulse, in particular in the case of aluminum.

\section{THE LOW CHARGE CASE}

A reduction in the electron bunch charge improves the FEL performance [3]. The electron beam brightness is increased due to the smaller spot size of the driver laser at the cathode of the rf photo-injector, reducing the contribution of the thermal emittance. It also allows for a more robust operation of the injector and linac, because, first, the impact of collective effects (e.g. CSR in the bunch compressor or wakefields in the rf structures) is reduced and, second, the energy chirp over the shorter bunch length becomes more linear and thus improves the efficiency of the bunch compressor.

With low charge, the current profile at the undulator entrance lacks the initial spike of the high charge case. This feature and the lower current of about $2.5 \mathrm{kA}$ reduce the transient in the wake potential by more than $50 \%$ (see Fig. 3). The shape of the wake potential is similar for both chamber materials and consists mainly out of one minimum. The length of the transient is comparable to the total bunch length, which then should yield a good efficiency in compensating the wakefields by an undulator field taper.

The GINGER results are shown in Fig. 4 and they verify the assumption of an improved performance for a lower bunch charge. The degradation of the FEL energy is $90 \%$ if no compensation for the wakefields is applied. However a field taper, which preserves the resonance condition for an energy loss of $200 \mathrm{keV} / \mathrm{m}$, completely recovers the FEL
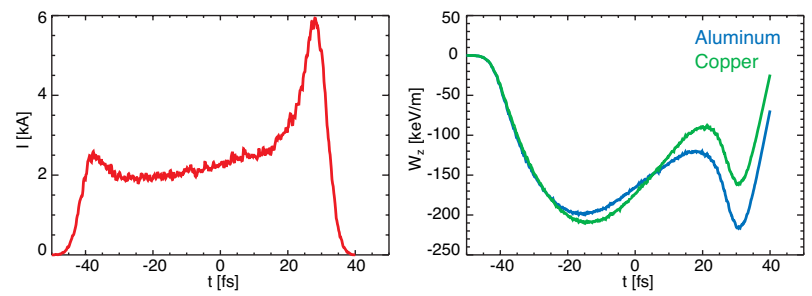

Figure 3: Current profile and undulator wakefields (left and right plot, respectively) for a $200 \mathrm{pC}$ electron bunch charge. 

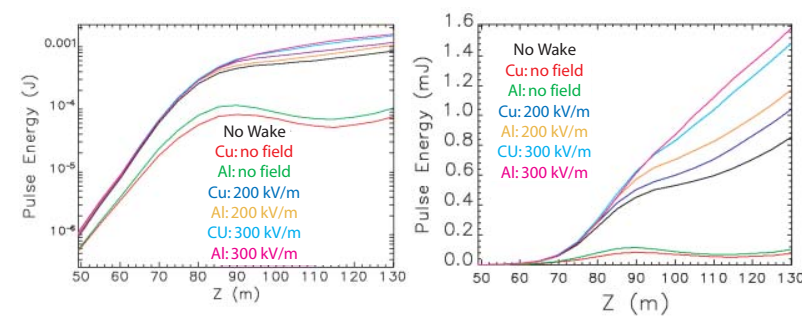

Figure 4: Ginger results for the $200 \mathrm{pC}$ case and various degrees of undulator field taper.

performance with a slight enhancement, compared to the low charge reference case which excludes all wakefields. The optimum performance is found for an equivalent field taper of $300 \mathrm{keV} / \mathrm{m}$. The energy of the radiation pulse is $1.6 \mathrm{~mJ}$, corresponding to $1.2 \cdot 10^{12}$ photons at a wavelength of 1.5 Angstrom, about twice as much as for the low charge reference case. This agrees with the optimal taper of 150 $\mathrm{keV} / \mathrm{m} \mathrm{[6]} \mathrm{to} \mathrm{double} \mathrm{the} \mathrm{output} \mathrm{power} \mathrm{of} \mathrm{the} \mathrm{FEL,} \mathrm{after} \mathrm{a}$ value of $150 \mathrm{keV} / \mathrm{m}$ has been subtracted to compensate for the average energy loss by the wakefields. The difference in the chamber material is negligible as expected from the similarity in the wake potential.

Another improvement in the FEL performance is that a compensating field taper avoids the fragmentation of the power envelope as it is the case for the $1 \mathrm{nC}$ bunch charge. This smooth profile is an essential requirement for advanced configuration such as chirped pulse amplification or pulse slicing [9].

\section{CODE COMPARISON}

The agreement between GINGER and GENESIS is quite good, except that GENESIS shows systematically a lower radiation energy than GINGER (see Fig. 5) by about $30 \%$. Relative parameters (e.g. gain length, saturation length or the efficiency of undulator field taper) are not affected by the difference in the absolute energy and therefore show excellent agreement. The difference may be explained by the fact that GINGER is a 2D code while GENESIS is 3D. Due to the different grid typologies grid points are weighted in the same manner in the codes even with the same grid spac-
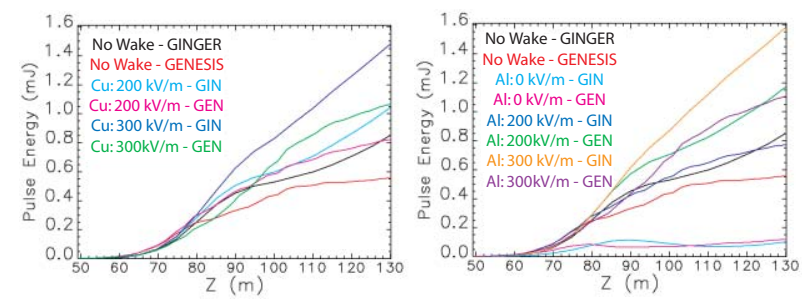

Figure 5: Comparison of the results by GINGER and GENESIS for the $200 \mathrm{pC}$ bunch charge case. ing. GENESIS supports a much larger number of modes that can couple with the electron beam and alter the FEL performance. In addition the reconstruction from the ELEGANT phase space output as well as the calculation of the effective wake potential could have yield slightly different results.

\section{CONCLUSIONS}

With the updated model for start-end simulation, including the frequency-dependence of the conductivity in the resistive wall wake fields, the FEL output energy is similar for both chamber materials at a bunch charge of $1 \mathrm{nC}$ and the optimized taper of the undulator field, with a slightly better performance for aluminum. The losses in the output energy by wakefields can be compensated by a taper in the undulator field. With optimum taper and aluminum as the choice for the chamber material the FEL pulse has an output energy of $2 \mathrm{~mJ}$, corresponding to $1.6 \cdot 10^{12}$ photons at $1.5 \AA$. Both materials yield a strong oscillation of the wake potential along the bunch, which causes the fragmentation of the FEL pulse into multiple sub-pulses. However this oscillation is damped stronger for aluminum with an almost constant value of the wake potential over the last part of the bunch. Therefore the pulse has fewer fragments if aluminum is used and the core of the electron bunch amplifies the spontaneous radiation more uniformly. This feature is essential for further pulse manipulations such as pulse slicing or compression.

Operating at a lower charge of $200 \mathrm{pC}$ improves not only the stability of the machine (see [3]) but reduces the undulator wakefields. The profile of the wake potential is similar for copper and aluminum and can be completely compensated by field taper. The FEL profile envelope is continuous and smooth. The photon count of $1.2 \cdot 10^{12}$ is only reduced by $25 \%$ for the low-charge case, as compared to a $80 \%$ reduction in the electron charge. For the sake of a stable operation and the processing of the FEL pulse (e.g. slicing of a chirped pulse) the low charge case is strongly preferred.

\section{ACKNOWLEDGMENT}

The work was supported by the DOE Contract No. DEAC02-76F00515

\section{REFERENCES}

[1] LCLS CDR, SLAC Report No. SLAC-R-593, 2002

[2] TESLA TDR, DESY Report No. DESY-2001-011,2001

[3] P. Emma et al., these PAC'05 proceedings

[4] S. Reiche et al., Nucl. Inst. \& Meth. A483 (2002) 70

[5] K. Bane and G. Stupakov, these PAC'05 proceedings

[6] Z. Huang and G. Stupakov, PRST-AB 8 (2005) 040702

[7] S. Reiche, LCLS-DOE Review Meeting, April 2004, SLAC

[8] H. Henke and O. Napoli, Proc. of the EPAC ' 90 conference, Nice, France

[9] C. Schroeder et al., J. Opt. Soc. Qm. B 19 (2002) 1782 